\title{
Matched cohort study of therapeutic strategies to prevent preschool wheezing/asthma attacks
}

This article was published in the following Dove Press journal: Journal of Asthma and Allergy

Jonathan Grigg,' Anjan

Nibber, ${ }^{2}$ James $Y$ Paton, ${ }^{3}$

Alison Chisholm, ${ }^{2}$ Theresa

W Guilbert, ${ }^{4}$ Alan Kaplan, ${ }^{5}$

Steve Turner, ${ }^{6}$ Nicolas

Roche, ${ }^{7}$ Elizabeth $\vee$ Hillyer, ${ }^{8}$

David B Price 8,9

On behalf of the

Respiratory Effectiveness

Group

'Blizard Institute, Queen Mary University of London, London, UK;

${ }^{2}$ Respiratory Effectiveness Group,

Cambridge, UK; ${ }^{3}$ School of Medicine,

College of Medical, Veterinary, and

Life Sciences, University of Glasgow,

Glasgow, UK; ${ }^{4}$ Pulmonary Division,

Cincinnati Children's Hospital

Medical Center, and Department of

Pediatrics, University of Cincinnati,

Cincinnati, OH, USA; ${ }^{5}$ Family Physician

Airways Group of Canada, University

of Toronto, Toronto, ON, Canada;

${ }^{6}$ Department of Child Health, Royal

Aberdeen Children's Hospital,

University of Aberdeen, Aberdeen,

UK; ${ }^{7}$ Respiratory Medicine, Cochin

Hospital Group, AP-HP, University

of Paris Descartes (EA25II), Paris,

France; ${ }^{8}$ Observational and Pragmatic

Research Institute Pte Ltd, Singapore,

Singapore; ${ }^{9}$ Academic Primary Care,

University of Aberdeen, Aberdeen, UK

Correspondence: Jonathan Grigg

Blizard Institute, Queen Mary University

of London, 4 Newark St., London EI

2AT, UK

Tel +44207882 2206

Email j.grigg@qmul.ac.uk
Background: An inhaled corticosteroid (ICS) or leukotriene receptor antagonist (LTRA) may prevent wheezing/asthma attacks in preschoolers with recurrent wheeze when added to shortacting $\beta$-agonist (SABA).

Objective: The aim of this historical matched cohort study was to assess the effectiveness of these treatments for preventing wheezing/asthma attacks.

Methods: Electronic medical records from the Optimum Patient Care Research Database were used to characterize a UK preschool population (1-5 years old) with two or more episodes of wheezing during 1 baseline year before first prescription (index date) of ICS or LTRA, or repeat prescription of SABA. Children initiating ICS or LTRA on the index date were matched 1:4 to those prescribed only SABA for age, sex, year of index prescription, mean baseline SABA dose, baseline attacks, baseline antibiotic prescriptions, and eczema diagnosis. Wheezing/ asthma attacks (defined as asthma-related emergency attendance, hospital admission, or acute oral corticosteroid prescription) during 1 outcome year were compared using conditional logistic regression.

Results: Matched ICS and SABA cohorts included 990 and 3,960 children, respectively (61\% male; mean [SD] age 3.2 [1.3] years), and matched LTRA and SABA cohorts included 259 and 1,036 children, respectively (65\% male; mean [SD] age 2.6 [1.2] years). We observed no significant difference between matched cohorts in the odds of a wheezing/asthma attack: ICS vs SABA, OR (95\% CI) 1.01 (0.85-1.19) and LTRA vs SABA, OR (95\% CI) 1.28 (0.96-1.72). Conclusion: We found no evidence that initiation of ICS or LTRA therapy is associated with fewer attacks during 1 outcome year than SABA alone for a heterogeneous group of preschool children with recurrent wheeze in the real-life clinical setting.

Keywords: electronic medical records, inhaled corticosteroids, leukotriene receptor antagonists, observational study, ICS particle size, short-acting $\beta$-agonist

\section{Introduction}

Acute episodes of asthma-like symptoms (wheezing/asthma attacks) are common in preschool children, affecting as many as one-third to one-half of children in the US and Western Europe at least once in their first 6 years. ${ }^{1-3}$ The associated health care burden is substantial, with children aged 1-5 years comprising the majority of children presenting to hospitals and emergency departments (EDs) in the US and UK with an attack of wheezing/asthma. ${ }^{4,5}$

The optimal approach for preventing recurrent episodes of wheezing/asthma in preschool children remains an active area of study and debate ${ }^{6-9}$ International and UK asthma management guidelines provide little definitive guidance for recurrent attack 
prevention in this age group. ${ }^{10,11}$ Wheezing phenotypes are heterogeneous and can change over time in an individual child. ${ }^{12,13}$ The results of landmark clinical trials indicate that for children at high risk of developing persistent asthma (ie, with positive asthma predictive index [API] $)^{14,15}$ who are asymptomatic between wheezing episodes, therapy with daily low-dose or intermittent (as needed) high-dose ICS reduces attack rate as well as symptom burden during attacks. ${ }^{16-18}$ More recently, a meta-analysis of randomized controlled trials (RCTs) found strong evidence to support intermittent ICS therapy for preschool children with intermittent asthma or viral-triggered wheeze. ${ }^{8}$ In addition, a recent study found no overall benefit of intermittent therapy with montelukast, a leukotriene receptor antagonist (LTRA), in preschool wheeze, albeit that need for rescue oral corticosteroid (OCS) (a secondary outcome) was reduced..$^{19}$ Overall, however, common conclusions from these studies are the marked heterogeneity of response and need for further studies. ${ }^{16-20}$

Studying preschool children is challenging both because of diagnostic and therapeutic limitations and because of the ethical issues and difficulties associated with enrolling sufficient numbers of children into clinical trials. ${ }^{21}$ Furthermore, strict trial eligibility criteria and the unique ecology of care provided in RCTs may limit the applicability of RCT results to the unselective and less controlled environment of everyday clinical practice, where frequent follow-up, extensive education of parents and children, and optimized adherence to therapy are often not feasible. ${ }^{22,23}$

Observational studies using anonymized medical record data can complement and supplement RCT results by enabling the study of large groups over long periods..$^{22,24}$ In a prior matched cohort study of children with asthma (ages 5-11) years, we found evidence of better effectiveness of extrafine (EF)-particle ICS (mass median aerodynamic diameter $[\mathrm{MMAD}]<2 \mu \mathrm{m}$ ) as compared with fine-particle ICS (MMAD $\geq 2 \mu \mathrm{m}){ }^{25}$ This led us to speculate that administration of an EFparticle ICS could also be more beneficial for the youngest children. Of note, almost all prior RCTs of preschool children used a fine-particle (rather than an EF-particle) ICS formulation, most commonly fluticasone propionate or budesonide. ${ }^{8}$

The aims of this historical matched cohort study were to compare outcomes for a broad general UK population of preschool children with evidence of prior wheezing who remained on either as-needed short-acting $\beta$-agonist (SABA) or stepped up to controller therapy (plus as-needed SABA), as either ICS or LTRA. In addition, we compared outcomes with the two controller therapies (ICS vs LTRA) and tested the hypothesis that use of EF-particle ICS would be associated with better outcomes than fine-particle ICS.

\section{Methods}

\section{Data source and study approval}

At the time of this study, the Optimum Patient Care Research Database contained anonymized, longitudinal medical record data for 2.5 million patients in the UK, where patients' electronic medical records, incorporating primary, secondary, and hospitalization care data, are centralized at their primary care practices. ${ }^{26}$ The study was conducted in compliance with all applicable local and international regulations and to standards suggested for observational studies (details in the Supplementary materials). ${ }^{27}$ The protocol, analyses, and dissemination of the results were approved by an independent steering committee of the Respiratory Effectiveness Group, ${ }^{28}$ a not-for-profit research and advocacy organization dedicated to furthering real-life clinical research.

\section{Study design and patients}

This was a matched cohort analysis employing a total of four two-way matched comparisons: 1) all ICSs by pressurized metered-dose inhaler (pMDI) vs SABA, 2) LTRA vs SABA, 3) LTRA vs all ICSs by pMDI, and 4) EF-particle ICS vs fine-particle ICS by pMDI. We studied two consecutive years of data for each child, including 1 baseline year for clinical characterization leading up to the index date, followed by 1 outcome year (Figure 1). The index date was defined as the time of either a first prescription for asthma controller therapy (listed below) or a repeat prescription for SABA.

Children eligible for the study were $\geq 1$ year and up to 5 years of age at the index date and during the baseline year had two or more wheezing episodes (described in diagnostic Read codes in the Supplementary materials) or two or more prescriptions (at different points in time) for any combination of OCS, with or without concomitant SABA, with accompanying code for a lower respiratory complaint. During the outcome year, children had to have at least one additional prescription for any asthma controller therapy (the same as or different from the index prescription) or two or more SABA prescriptions (SABA control arms).

Children $<1$ year old were excluded to ensure 1 full year of baseline data. Other exclusion criteria were any chronic respiratory disease other than wheeze/asthma, ICS or LTRA prescription during the baseline year, combination LABAICS prescription during the baseline year or on the index date, or multiple step-up therapies on the index date. The study period was from January 1988 to May 2015.

Asthma controller therapies included as index date prescriptions were EF-particle ICS delivered by pMDI (ciclesonide or EF-particle beclomethasone [Qvar ${ }^{\circledR}$; Teva UK Ltd., Castleford, UK]), fine-particle ICS delivered by pMDI 


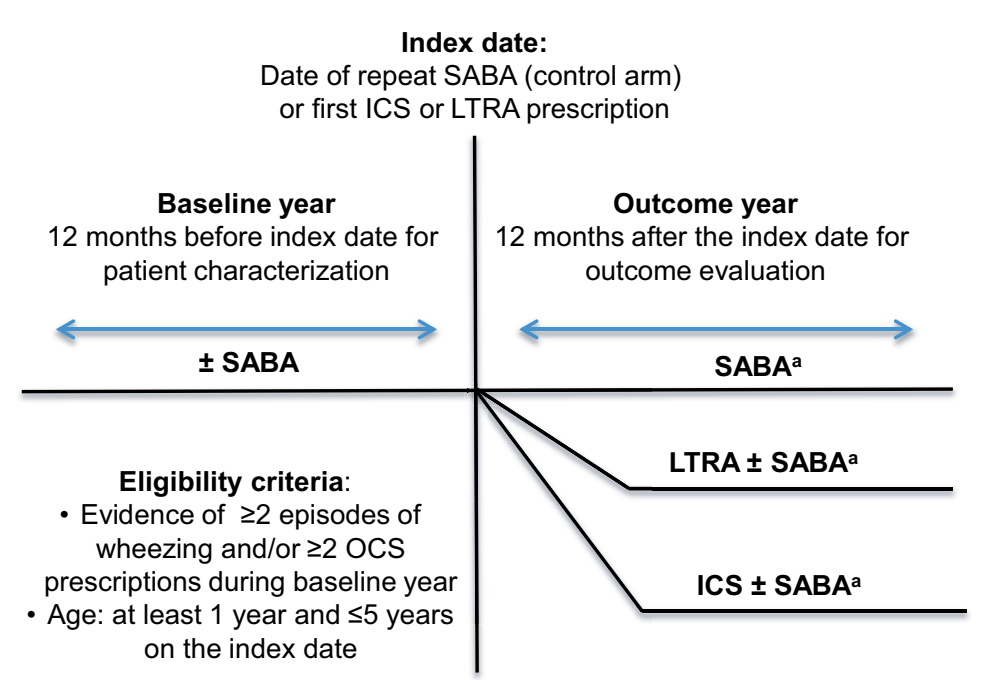

Figure I Study schematic.

Notes: The eligibility criterion of two or more OCS prescriptions (at different points in time) could be for any combination of OCS, with or without concomitant SABA, with accompanying code for a lower respiratory complaint. aPrescribed therapy could change during the outcome year after the index date.

Abbreviations: ICS, inhaled corticosteroid; LTRA, leukotriene receptor antagonist; OCS, oral corticosteroid; SABA, short-acting $\beta$-agonist.

(fluticasone, budesonide, or fine-particle beclomethasone [Clenil ${ }^{\circledR}$ Modulite $^{\circledR}$; Chiesi Ltd., Manchester, UK]), and LTRAs. Children belonging to the SABA arms (but not those in controller therapy arms) were required to have at least one SABA prescription during the baseline year before the index date; the index date SABA prescription for the study was identified through the matching process as providing the best match. Therapy could change during the outcome year as prescribed by the health care provider.

\section{Outcome measures}

The primary study end point was a wheezing/asthma attack, defined according to the American Thoracic Society/ European Respiratory Society criteria for a severe asthma exacerbation as any of the following: an asthma-related ED attendance, an asthma-related hospital admission, or an OCS prescription coded for asthma or wheeze. ${ }^{29}$ We note that this measure differed from the baseline year wheezing episodes applied as a study eligibility criterion, which we defined according to diagnostic Read codes (Supplementary materials) and for which the definition did not necessarily include an asthma-related ED attendance, hospital admission, or OCS prescription.

Secondary end points, defined in Table S1, included acute respiratory events, risk-domain asthma control, and treatment stability, composite end points utilized in prior studies by the authors. ${ }^{25,30,31}$ Exploratory outcome measures included oral candidiasis (thrush) and time to first wheezing/ asthma attack.
We calculated daily SABA dose by averaging the number of doses in issued prescriptions during each study year, with one dose defined as two puffs $(200 \mu \mathrm{g})$. Similarly, the average daily ICS dose exposure during the outcome year was calculated using the total ICS dose in prescriptions during the outcome year divided by 365 days. The doses of ICS were standardized to equivalence with fluticasone propionate; thus, the actual doses of fluticasone and EF-particle beclomethasone were used, and doses of budesonide and fine-particle beclomethasone were halved.

\section{Statistical analysis}

Children in each set of two comparison cohorts were matched, at ratios selected to maximize statistical power, sequentially for the following key demographic and baseline year clinical characteristics (details in the Supplementary materials): age (years) at the index date, sex, index date year, mean daily SABA doses, wheezing/asthma attacks (yes/no), acute antibiotic prescriptions (yes/no), and ever-recorded eczema (yes/no).

The four sets of two matched cohorts were compared for baseline characteristics and binary outcome measures using conditional logistic regression. Kaplan-Meier curves for time to first wheezing/asthma attack were produced for each matched cohort comparison, and the time to first attack was compared using a stratified log-rank test for equality of survivor curves across matched cohorts. Children who experienced treatment failure before a first wheezing/asthma attack were censored at 7 days after the date of treatment failure, 
defined as addition of new therapy or $\geq 50 \%$ increase in dose of index date therapy. Treatment failure was included as a variable only in the Kaplan-Meier analyses (further information is provided in the Supplementary materials).

Matching was completed using SAS version 9.3 (SAS Institute, Cary, NC, USA). Outcome analyses were carried out using Stata version 14.1 (StataCorp LLC, College Station, TX, USA).

\section{Results}

\section{Patients}

Children who met eligibility criteria numbered 11,329 in the ICS cohort, 335 in the LTRA cohort, and 12,373 in the SABA cohort. Figures S1-S4 depict the numbers of children (or medical records in the case of SABA) retained at each step of the matching process for the four two-way comparisons.

\section{Controller therapy versus SABA}

\section{Patients}

The ICS and LTRA cohorts were each matched in 1:4 ratio to the SABA cohort, resulting in 990:3,960 (ICS:SABA) and 259:1,036 (LTRA:SABA) children in the two analyses (Figures S1 and S2). In the ICS vs SABA comparison, 61\% of children were male and the mean age was 3.2 years; in the LTRA vs SABA comparison, $65 \%$ of children were male and the mean age was 2.6 years. The prevalence of recorded eczema was $38 \%$ in each of the four cohorts (Table 1 ).

Overall, $36 \%$ of children in the matched ICS/SABA cohorts had experienced one or more wheezing/asthma

Table I Baseline year characteristics of children included in the controller (ICS or LTRA) vs SABA matched cohort comparisons

\begin{tabular}{|c|c|c|c|c|}
\hline \multirow[t]{2}{*}{ Characteristics } & \multicolumn{2}{|l|}{ ICS vs SABA } & \multicolumn{2}{|l|}{ LTRA vs SABA } \\
\hline & $\operatorname{ICS}(n=990)$ & SABA $(n=3,960)$ & LTRA $(n=259)$ & SABA $(n=1,036)$ \\
\hline Male sex, n (\%) ${ }^{\mathrm{a}}$ & $602(60.8)$ & $2,408(60.8)$ & $168(64.9)$ & $672(64.9)$ \\
\hline Age at index date (years), mean (SD) ${ }^{\mathrm{a}}$ & $3.2(1.3)$ & $3.2(1.3)$ & $2.6(1.2)$ & $2.6(1.2)$ \\
\hline \multicolumn{5}{|l|}{ Comorbidity, n (\%) } \\
\hline Eczema $^{\mathrm{a}}$ & $372(37.6)$ & $\mathrm{I}, 488(37.6)$ & $97(37.5)$ & $388(37.5)$ \\
\hline Rhinitis & $36(3.6)$ & $170(4.3)$ & II (4.3) & $35(3.4)$ \\
\hline \multicolumn{5}{|l|}{ Total wheezing/asthma attacks, $\mathrm{n}(\%)^{\mathrm{a}}$} \\
\hline 0 & $635(64.1)$ & $2,540(64.1)$ & I34 (5 I.7) & $536(5 \mathrm{I} .7)$ \\
\hline$\geq 1$ & $355(35.9)$ & $\mathrm{I}, 420(35.9)$ & $125(48.3)$ & $500(48.3)$ \\
\hline \multicolumn{5}{|l|}{ OCS courses, n (\%) } \\
\hline 0 & $656(66.3)$ & $2,657(67.1)$ & $147(56.8)$ & $582(56.2)$ \\
\hline I & $222(22.4)$ & $884(22.3)$ & $59(22.8)$ & $293(28.3)$ \\
\hline 2 & 78 (7.9) & $320(8.1)$ & $30(11.6)$ & II 5 (II.I) \\
\hline$\geq 3$ & $34(3.4)$ & $99(2.5)$ & $10(3.9)$ & $9(0.9)$ \\
\hline$\geq I$ wheezing/asthma attack and $\geq I$ acute OCS course & $334(33.7)$ & $1,303(32.9)$ & $112(43.2)$ & $454(43.8)$ \\
\hline \multicolumn{5}{|l|}{ Acute antibiotic prescriptions, $\mathrm{n}(\%)^{\mathrm{a}}$} \\
\hline 0 & $510(51.5)$ & $2,040(51.5)$ & $105(40.5)$ & $420(40.5)$ \\
\hline 1 & $235(23.7)$ & $981(24.8)$ & $59(22.8)$ & $293(28.3)$ \\
\hline 2 & $129(13.0)$ & $539(13.6)$ & $54(20.9)$ & $180(17.4)$ \\
\hline$\geq 3$ & $116(11.7)$ & $400(10.1)$ & $21(8.1)$ & $58(5.6)$ \\
\hline Mean (SD) daily no. of SABA doses ${ }^{\mathrm{a}, \mathrm{c}}$ & $0.65(0.56)$ & $0.65(0.47)$ & $0.66(0.50)$ & $0.66(0.49)$ \\
\hline Median (IQR) daily no. of SABA doses ${ }^{c}$ & $0.55(0.55-0.82)$ & $0.55(0.55-0.82)$ & $0.55(0.55-0.82)$ & $0.55(0.55-0.82)$ \\
\hline Hospital admission, $\geq \mathrm{I}, \mathrm{n}(\%)$ & $36(3.7)$ & $157(4.0)$ & $21(8.1)$ & $64(6.2)^{\mathrm{e}}$ \\
\hline ED attendance, $\geq \mathrm{I}, \mathrm{n}(\%)$ & $13(1.3)$ & $52(1.3)$ & $19(7.3)$ & $20(2.0)$ \\
\hline Acute respiratory event, $\geq 1, n(\%)^{d}$ & $617(62.3)$ & $2,468(62.3)$ & $193(74.5)$ & $772(74.5)$ \\
\hline Risk-domain asthma control, $\mathrm{n}(\%)^{d}$ & $373(37.7)$ & $\mathrm{I}, 492(37.7)$ & $66(25.5)$ & $264(25.5)$ \\
\hline Oral candidiasis, $\geq \mathrm{I}, \mathrm{n}(\%)^{\mathrm{d}}$ & II (I.I) & $55(1.4)$ & $7(2.7)$ & $26(2.5)$ \\
\hline Pneumonia, yes, n (\%) ${ }^{d}$ & $12(1.2)$ & $43(1.1)$ & $9(3.5)$ & $8(0.8)^{\mathrm{e}}$ \\
\hline
\end{tabular}

Notes: a Matching variable. Wheezing/asthma attacks were defined as any of the following: an asthma-related ED attendance, an asthma-related hospital admission, or an OCS prescription coded for asthma or wheeze. ' ${ }^{\circ}$ Comorbidities were defined as follows: eczema as ever-recorded diagnostic Read code + topical corticosteroid; and rhinitis, as ever-recorded diagnosis and/or prescription for nasal corticosteroids. 'The daily SABA dose was calculated as the number of doses in issued prescriptions averaged over the baseline year. One SABA dose was two puffs ( $100 \mu \mathrm{g}$ per puff). ${ }^{\mathrm{d} A n}$ acute respiratory event was defined as occurrence of $\left.\mathrm{I}\right)$ an asthma-related hospital admission or ED attendance or 2) an acute course of OCSs coded for asthma or 3) antibiotics prescribed with a lower respiratory consultation. Risk-domain asthma control was defined as follows: I) no asthma-related hospital admission, ED attendance, or outpatient department attendance; 2) no acute OCS prescription with a lower respiratory consultation; and 3) no antibiotics prescribed with a lower respiratory consultation (Table SI). Oral candidiasis (thrush) was defined as a Read code for oral candidiasis or topical antifungal prescription definitely for treating oral candidiasis. Pneumonia was defined as a diagnostic Read code for pneumonia. ${ }^{e} \leq 0.00 \mathrm{I}$ for comparison between LTRA and SABA cohorts. There were no significant differences between ICS and SABA cohorts or between LTRA and SABA cohorts for other variables.

Abbreviations: ED, emergency department; ICS, inhaled corticosteroid; IQR, interquartile range; LTRA, leukotriene receptor antagonist; OCS, oral corticosteroid; SABA, short-acting $\beta$-agonist. 
attacks during the baseline year. In the matched LTRA/ SABA cohorts, $48 \%$ of children had experienced one or more attacks during the baseline year, including $24 \%$ and $17 \%$ in LTRA and SABA cohorts, respectively, who had experienced two or more attacks. Approximately half of children in the matched ICS/SABA cohorts and $60 \%$ of children in the matched LTRA/SABA cohorts had received one or more antibiotic prescriptions during the baseline year (Table 1).
The median (IQR) dose of ICS prescribed on the index date was fluticasone-equivalent 100 (100-200) $\mu \mathrm{g} /$ day (budesonide-equivalent dose, 200 [200-400] $\mu \mathrm{g} /$ day).

\section{Outcomes}

There was no significant difference in the odds of a wheezing/ asthma attack during the outcome year between the matched cohorts in either of the two comparisons between controller therapy and SABA (Figure 2; Table 2). During the outcome

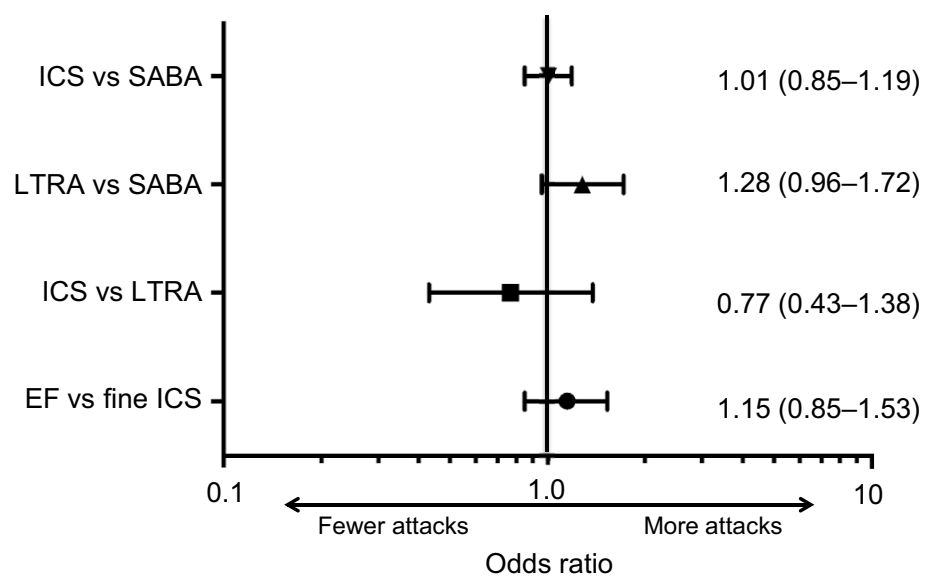

Figure 2 Forest plot depicting OR $(95 \% \mathrm{Cls})$ of wheezing/asthma attack for the four matched cohort comparisons.

Notes: The reference cohort (attack odds $=1.0$ ) is listed second. EF: EF particle; fine: fine particle.

Abbreviations: EF, extrafine; ICS, inhaled corticosteroid; LTRA, leukotriene receptor antagonist; SABA, short-acting $\beta$-agonist.

Table 2 Outcome measures during I follow-up year for the controller (ICS or LTRA) vs SABA matched cohort comparisons

\begin{tabular}{|c|c|c|c|c|c|c|}
\hline \multirow[t]{2}{*}{ Outcome } & \multicolumn{3}{|l|}{ ICS vs SABA } & \multicolumn{3}{|l|}{ LTRA vs SABA } \\
\hline & ICS $(n=990)$ & SABA $(n=3,960)$ & $P$-value ${ }^{a}$ & LTRA $(n=259)$ & SABA $(n=1,036)$ & $P$-value ${ }^{a}$ \\
\hline \multicolumn{7}{|c|}{ Wheezing/asthma attack, n (\%) } \\
\hline 0 & $728(73.5)$ & $2,918(73.7)$ & 0.92 & $157(60.6)$ & $682(65.8)$ & 0.097 \\
\hline 1 & I6I (16.3) & $649(16.4)$ & & $54(20.9)$ & $216(20.9)$ & \\
\hline$\geq 2$ & $101(10.2)$ & $393(9.9)$ & & $48(18.5)$ & $138(13.3)$ & \\
\hline \multicolumn{7}{|c|}{ Hospital admission, $\mathrm{n}(\%)$} \\
\hline 0 & $975(98.5)$ & $3,884(98.1)$ & 0.40 & $25 I(96.9)$ & $\mathrm{I}, 002(96.7)$ & 0.88 \\
\hline 1 & $12(1.2)$ & 67 (1.7) & & $6(2.3)$ & $28(2.7)$ & \\
\hline$\geq 2$ & $3(0.3)$ & $9(0.2)$ & & $2(0.8)$ & $6(0.6)$ & \\
\hline \multicolumn{7}{|c|}{ ED visit, $\mathrm{n}(\%)$} \\
\hline 0 & $969(97.9)$ & $3,921(99.0)$ & 0.004 & $252(97.3)$ & $\mathrm{I}, 022(98.7)$ & 0.13 \\
\hline I & $19(1.9)$ & $37(0.9)$ & & $5(1.9)$ & $12(1.2)$ & \\
\hline 2 & $0(0)$ & $2(0.1)$ & & $2(0.8)$ & $2(0.2)$ & \\
\hline 3 & $2(0.2)$ & $0(0)$ & & 0 & 0 & \\
\hline \multicolumn{7}{|c|}{ Acute OCS prescription, n (\%) } \\
\hline 0 & $814(82.2)$ & $3,250(82.1)$ & 0.91 & $164(63.3)$ & $703(67.9)$ & 0.14 \\
\hline I & $128(12.9)$ & $509(12.9)$ & & $46(17.8)$ & $206(19.9)$ & \\
\hline 2 & $33(3.3)$ & $144(3.6)$ & & $23(8.9)$ & 77 (7.4) & \\
\hline 3 & $8(0.8)$ & 45 (I.I) & & 0 & 0 & \\
\hline 4 & $6(0.6)$ & $10(0.3)$ & & $4(1.5)$ & $9(0.9)$ & \\
\hline$\geq 5$ & $\mathrm{I}(0.1)$ & I (0.0) & & $3(1.2)$ & 0 & \\
\hline
\end{tabular}

Note: a Matched cohorts were compared (for none vs $\geq 1$ outcome) using conditional logistic regression.

Abbreviations: ED, emergency department; ICS, inhaled corticosteroid; LTRA, leukotriene receptor antagonist; OCS, oral corticosteroid; SABA, short-acting $\beta$-agonist. 
year, $26 \%$ of children in the matched ICS/SABA cohorts experienced one or more attacks; and in the matched LTRA/ SABA comparison, $39 \%$ and $34 \%$ of children in LTRA and SABA cohorts, respectively, experienced one or more attacks (Figure 3; Table 2). Fewer children experienced attacks during the outcome than the baseline year (Figure 3).

A significantly higher percentage of children in the ICS cohort $(2.1 \%)$ vs the matched SABA cohort $(1.0 \%)$ experienced one or more $\mathrm{ED}$ visits $(P=0.004)$. There were no other significant differences between the two sets of matched cohorts in secondary outcome measures (Tables 2 and S2).

In the matched ICS/SABA comparison, 1,986 (50\%) children in the SABA cohort were prescribed ICS at some point during the outcome year. In the matched LTRA/SABA comparison, $162(63 \%)$ and $521(50 \%)$ children in LTRA and
SABA cohorts, respectively, were prescribed ICS during the outcome year.

The time to first wheezing/asthma attack was similar in the two comparisons (log-rank $P>0.10$; Figures S5 and S6).

\section{Comparisons of controller therapies}

\section{Patients}

The LTRA and ICS cohorts were matched 1:1, resulting in 104 children in each cohort (Figure S3); the EF-particle ICS and fine-particle ICS cohorts were matched 1:4, resulting in 275 and 1,100 children in each cohort, respectively (Figure S4). In the matched LTRA/ICS comparison, 61\% of children were male and mean age was 2.6 years; in the matched EF-particle ICS/fine-particle ICS comparison, $68 \%$ of children were male and mean age was 3.2 years (Table 3 ).
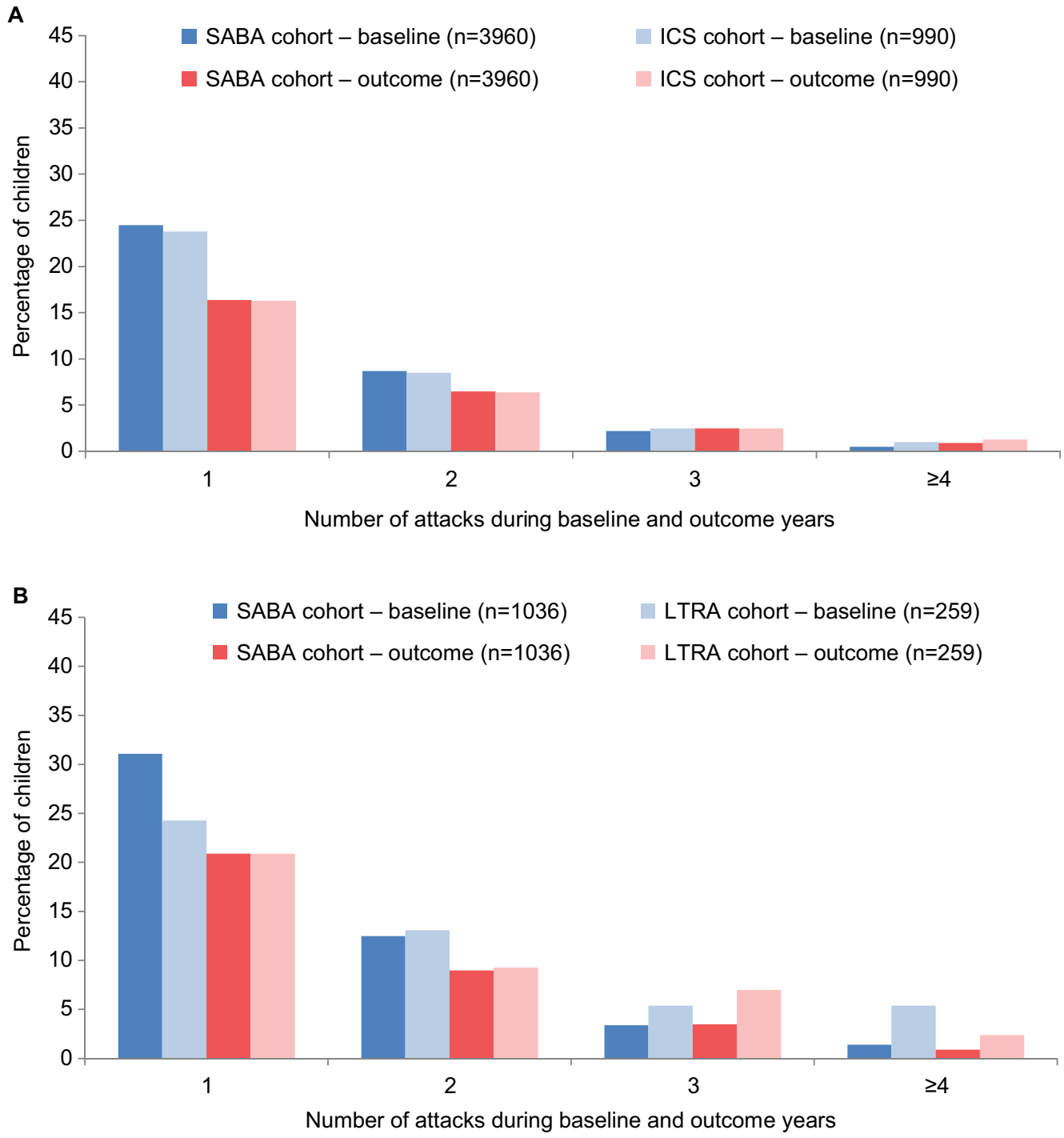

Figure 3 (Continued) 

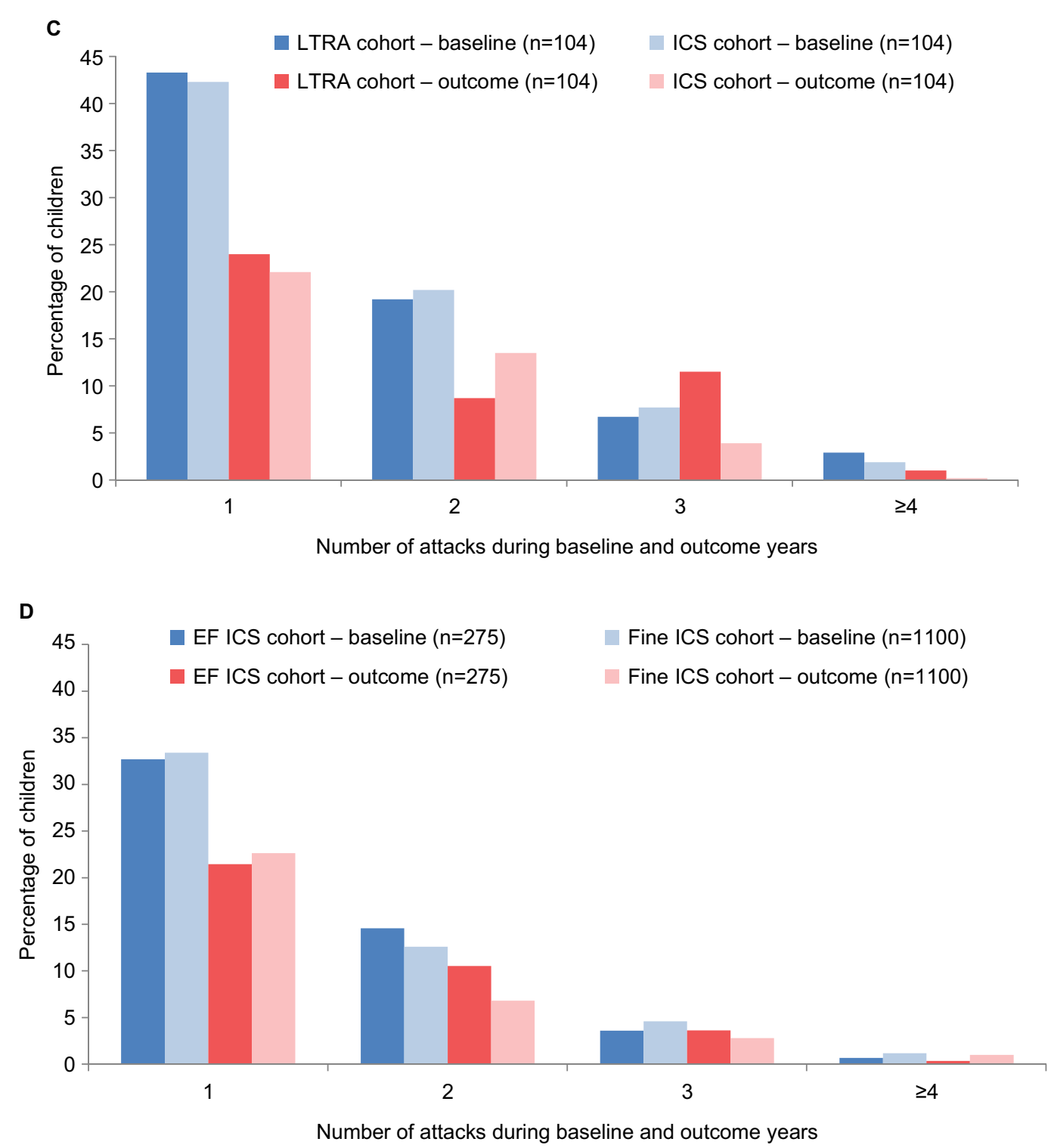

Figure 3 Percentage of children with one or more wheezing/asthma attacks during the baseline year (before the first prescription of ICS, LTRA, or repeat SABA) and during the outcome year in the four matched cohort comparisons: (A) ICS \pm SABA vs SABA, (B) LTRA \pm SABA vs SABA, (C) LTRA vs ICS, and (D) EF-particle ICS vs fine-particle ICS.

Notes: A wheezing/asthma attack was defined as an asthma-related ED attendance, an asthma-related hospital admission, or an OCS prescription coded for asthma or wheeze. EF: EF particle; fine: fine particle.

Abbreviations: ED, emergency department; EF, extrafine; ICS, inhaled corticosteroid; LTRA, leukotriene receptor antagonist; OCS, oral corticosteroid; SABA, short-acting $\beta$-agonist.

The prevalence of recorded eczema was $38 \%$ in matched LTRA/ICS cohorts and $41 \%$ in matched EF-particle ICS/ fine-particle ICS cohorts.

During the baseline year, one or more wheezing/asthma attacks were recorded for $72 \%$ of children in the matched LTRA/ICS cohorts and $52 \%$ of children in the matched EFparticle ICS/fine-particle ICS cohorts (Table 3). Approximately $71 \%$ of children in matched LTRA/ICS cohorts and $55 \%$ of children in matched EF-particle ICS/fine-particle ICS cohorts had received one or more antibiotic prescriptions during the baseline year (Table 3).
On the index date, the prescribed median (IQR) fluticasone-equivalent dose was $100(100-100) \mu \mathrm{g} /$ day in the ICS cohort of the matched LTRA/ICS comparison and 100 (100-100) and $200(100-200) \mu \mathrm{g} /$ day in matched EF-particle ICS and fine-particle ICS cohorts, respectively.

\section{Outcomes}

There was no significant difference in the odds of a wheezing/ asthma attack during the outcome year between the matched cohorts in either of the two controller therapy comparisons (Figure 2; Table 4). In the matched LTRA/ICS cohorts, $45 \%$ 
Table 3 Baseline year characteristics of children included in the two controller therapy matched cohort comparisons: LTRA vs ICS and EF-particle ICS vs fine-particle ICS

\begin{tabular}{|c|c|c|c|c|}
\hline \multirow[t]{2}{*}{ Characteristics } & \multicolumn{2}{|l|}{ LTRA vs ICS } & \multicolumn{2}{|c|}{ EF-particle ICS vs fine-particle ICS } \\
\hline & $\begin{array}{l}\text { LTRA } \\
(n=104)\end{array}$ & $\begin{array}{l}\text { ICS } \\
(n=104)\end{array}$ & $\begin{array}{l}\text { EF-particle ICS } \\
(n=275)\end{array}$ & $\begin{array}{l}\text { Fine-particle ICS } \\
(n=I, 100)\end{array}$ \\
\hline Male sex, n (\%) ${ }^{\mathrm{a}}$ & $63(60.6)$ & $63(60.6)$ & $186(67.6)$ & $744(67.6)$ \\
\hline Age at index date (years), mean (SD) ${ }^{\mathrm{a}}$ & $2.6(I . I)$ & $2.6(I . I)$ & $3.2(1.2)$ & $3.2(1.2)$ \\
\hline \multicolumn{5}{|l|}{ Comorbidity, n (\%) ${ }^{b}$} \\
\hline Eczema $^{a}$ & $39(37.5)$ & $39(37.5)$ & $113(4 \mid .1)$ & $452(4 I . I)$ \\
\hline Rhinitis & $6(5.8)$ & $7(6.7)$ & $10(3.6)$ & $4 I(3.7)$ \\
\hline \multicolumn{5}{|l|}{ Total wheezing/asthma attacks, $\mathrm{n}(\%)^{\mathrm{a}}$} \\
\hline 0 & $29(27.9)$ & $29(27.9)$ & $133(48.4)$ & $532(48.4)$ \\
\hline$\geq 1$ & $75(72.1)$ & $75(72.1)$ & $142(5 \mid .6)$ & $568(5 \mathrm{I} .6)$ \\
\hline \multicolumn{5}{|l|}{ OCS courses, n (\%) } \\
\hline 0 & $35(33.7)$ & $32(30.8)$ & $140(50.9)$ & $565(5 \mathrm{I} .4)$ \\
\hline I & $41(39.4)$ & $4 \mathrm{l}(39.4)$ & $86(31.3)$ & $340(30.9)$ \\
\hline 2 & $20(19.2)$ & $20(19.2)$ & $36(13.1)$ & $128(11.6)$ \\
\hline$\geq 3$ & $8(7.7)$ & II (10.6) & $13(4.7)$ & $67(6.1)$ \\
\hline $\begin{array}{l}\geq I \text { wheezing/asthma attack and } \geq I \\
\text { acute OCS course }\end{array}$ & $69(66.3)$ & $72(69.2)$ & $135(49.1)$ & $535(48.6)$ \\
\hline \multicolumn{5}{|l|}{ Acute antibiotic prescriptions, $\mathrm{n}(\%)^{\mathrm{a}}$} \\
\hline 0 & $30(28.9)$ & $30(28.9)$ & $123(44.7)$ & $492(44.7)$ \\
\hline I & $33(31.7)$ & $29(27.9)$ & $72(26.2)$ & $280(25.5)$ \\
\hline 2 & $20(19.2)$ & $16(15.4)$ & $49(17.8)$ & $183(16.6)$ \\
\hline$\geq 3$ & $21(20.2)$ & $29(27.9)$ & $31(11.3)$ & $145(13.2)$ \\
\hline Mean (SD) daily no. of SABA doses ${ }^{a, c}$ & $0.47(0.27)$ & $0.47(0.27)$ & $0.63(0.4)$ & $0.63(0.4)$ \\
\hline Median (IQR) daily no. of SABA doses ${ }^{c}$ & $0.55(0.27-0.55)$ & $0.55(0.27-0.55)$ & $0.55(0.27-0.82)$ & $0.55(0.27-0.82)$ \\
\hline Hospital admission, $\geq \mathrm{I}, \mathrm{n}(\%)$ & $12(11.5)$ & $7(6.7)$ & $14(5.1)$ & $47(4.3)$ \\
\hline ED attendance, $\geq \mathrm{I}, \mathrm{n}(\%)$ & $9(8.7)$ & $2(1.9)^{\mathrm{e}}$ & $5(1.8)$ & $25(2.2)$ \\
\hline Acute respiratory event, $\geq 1, n(\%)^{d}$ & $89(85.6)$ & $89(85.6)$ & $142(5 \mid .6)$ & $568(51.6)$ \\
\hline 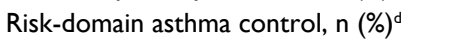 & $15(14.4)$ & $15(14.4)$ & $68(24.5)$ & $272(24.7)$ \\
\hline Oral candidiasis, $\geq \mathbf{I}, \mathrm{n}(\%)^{\mathrm{d}}$ & $3(2.9)$ & 0 & $2(0.7)$ & $14(1.3)$ \\
\hline Pneumonia, yes, $n(\%)^{d}$ & $4(3.8)$ & $\mathrm{I}(\mathrm{I} .0)$ & $2(0.7)$ & $8(0.7)$ \\
\hline
\end{tabular}

Notes: ${ }^{a}$ Matching variable. ${ }^{b}$ Comorbidities were defined as follows: eczema as ever-recorded diagnostic Read code + topical steroid; and rhinitis as ever-recorded diagnosis and/or prescription for nasal steroids. 'The daily SABA dose was calculated as the number of doses in issued prescriptions averaged over the baseline year. One SABA dose was two puffs ( $100 \mu \mathrm{g}$ per puff). ${ }^{\mathrm{d}} \mathrm{An}$ acute respiratory event was defined as occurrence of I) an asthma-related hospital admission or ED attendance or 2 ) an acute course of OCSs coded for asthma or 3) antibiotics prescribed with a lower respiratory consultation. Risk-domain asthma control was defined as follows: I) no asthma-related hospital admission, ED attendance, or outpatient department attendance; 2) no acute OCS prescription with a lower respiratory consultation; and 3) no antibiotics prescribed with a lower respiratory consultation. Oral candidiasis (thrush) was defined as a Read code for oral candidiasis or topical antifungal prescription definitely for treating oral candidiasis. Pneumonia was defined as a diagnostic Read code for pneumonia. ${ }^{e}=0.03$ for comparison between LTRA and ICS cohorts. There were no significant differences between EF-particle ICS and fine-particle ICS cohorts or between LTRA and ICS cohorts for other variables.

Abbreviations: ED, emergency department; EF, extrafine; ICS, inhaled corticosteroid; IQR, interquartile range; LTRA, leukotriene receptor antagonist; OCS, oral corticosteroid; SABA, short-acting $\beta$-agonist.

and $39 \%$ of children, respectively, experienced one or more attacks; in the matched EF-particle ICS/fine-particle ICS cohorts, $36 \%$ and $33 \%$ of children, respectively, experienced one or more attacks (Figure 3; Table 4). Overall, fewer children experienced attacks during the outcome than the baseline year (Figure 3).

There were no statistically significant differences between matched cohorts for either of the two controller therapy comparisons during the outcome year (Tables 4 and S3).

Seventy-two (69\%) children in the LTRA cohort were prescribed ICS and eight (8\%) children in the ICS cohort were prescribed LTRA during the outcome year.
The times to first wheezing/asthma attack were similar in the two comparisons (log-rank $P \geq 0.10$; Figures $\mathrm{S} 7$ and $\mathrm{S} 8$ ).

\section{Discussion}

We found no significant differences in the odds of wheezing/ asthma attacks during the outcome year in any of the four treatment comparisons, including ICS vs SABA, LTRA vs SABA, LTRA vs ICS, and EF-particle ICS vs fine-particle ICS, between matched cohorts of preschool children with two or more prior episodes of wheezing and/or OCS prescriptions. The only statistically significant difference we observed during the outcome year is perhaps not clinically significant in 
Table 4 Outcome measures during I follow-up year for the controller therapy matched cohort comparisons: LTRA vs ICS and EFparticle ICS vs fine-particle ICS

\begin{tabular}{|c|c|c|c|c|c|c|}
\hline \multirow[t]{2}{*}{ Outcome } & \multicolumn{3}{|l|}{ LTRA vs ICS } & \multicolumn{3}{|c|}{ EF-particle ICS vs fine-particle ICS } \\
\hline & LTRA $(n=104)$ & ICS $(n=104)$ & $P$-value ${ }^{a}$ & $\begin{array}{l}\text { EF-particle ICS } \\
(n=275)\end{array}$ & $\begin{array}{l}\text { Fine-particle ICS } \\
(n=I, 100)\end{array}$ & $P$-value ${ }^{a}$ \\
\hline \multicolumn{7}{|c|}{ Wheezing/asthma attack, n (\%) } \\
\hline 0 & $57(54.8)$ & $63(60.6)$ & 0.38 & $176(64.0)$ & $734(66.7)$ & 0.36 \\
\hline $\mathrm{I}$ & $25(24.0)$ & $23(22.1)$ & & $59(21.5)$ & $249(22.6)$ & \\
\hline$\geq 2$ & $22(21.2)$ & $18(17.3)$ & & $40(14.5)$ & $117(10.6)$ & \\
\hline \multicolumn{7}{|c|}{ Hospital admission, n (\%) } \\
\hline 0 & $101(97.1)$ & $102(98.1)$ & 0.66 & $268(97.5)$ & $\mathrm{I}, 075(97.7)$ & 0.79 \\
\hline 1 & $2(1.9)$ & $\mathrm{I}(\mathrm{I} .0)$ & & $7(2.5)$ & $22(2.0)$ & \\
\hline 2 & $\mathrm{I}(\mathrm{I} .0)$ & $\mathrm{I}(\mathrm{I} .0)$ & & 0 & $3(0.3)$ & \\
\hline \multicolumn{7}{|c|}{ ED visit, $\mathrm{n}(\%)$} \\
\hline 0 & $100(96.2)$ & $103(99.0)$ & 0.22 & $27 \mid(98.6)$ & $\mathrm{I}, 087(98.8)$ & 0.71 \\
\hline I & $4(3.8)$ & $\mathrm{I}(\mathrm{I} .0)$ & & $4(1.4)$ & $14(1.0)$ & \\
\hline 2 & 0 & 0 & & 0 & 0 & \\
\hline 3 & 0 & 0 & & 0 & $2(0.2)$ & \\
\hline \multicolumn{7}{|c|}{ Acute OCS prescription, $\mathrm{n}(\%)$} \\
\hline 0 & $60(57.7)$ & $64(61.5)$ & 0.78 & $204(74.2)$ & $846(76.9)$ & 0.32 \\
\hline 1 & $21(20.2)$ & $20(19.2)$ & & $48(17.5)$ & $182(16.6)$ & \\
\hline 2 & $11(10.6)$ & $15(14.4)$ & & $19(6.9)$ & $51(4.6)$ & \\
\hline 3 & II (10.6) & $4(3.9)$ & & $4(1.5)$ & $12(1.1)$ & \\
\hline 4 & $I(I .0)$ & $I(1.0)$ & & 0 & $8(0.7)$ & \\
\hline 5 & 0 & 0 & & 0 & $I(0.1)$ & \\
\hline
\end{tabular}

Note: a Matched cohorts were compared (for none vs $\geq 1$ outcome) using conditional logistic regression.

Abbreviations: ED, emergency department; EF, extrafine; ICS, inhaled corticosteroid; LTRA, leukotriene receptor antagonist; OCS, oral corticosteroid.

light of other findings and the few children affected, namely, $2 \%$ vs $1 \%$ of children with an ED visit in the ICS vs SABA cohorts, respectively. These results contrast with those of our previous study of older children, for whom there were indications of better effectiveness of EF-particle ICS than fine-particle ICS, ${ }^{25}$ as we had found in prior adult studies. ${ }^{30-32}$

One possible explanation for our findings is that there is genuinely no effect of any of these agents (EF-particle ICS, fine-particle ICS, or LTRA) with regard to preventing wheezing/asthma attacks in preschool children with intermittent disease. Several other explanations are possible, however. The mix of asthma phenotypes among preschool children in our large, general asthma population may preclude identifying a single effective agent. Indeed, this heterogeneity could explain the negative results of this study, including both the heterogeneity of preschool wheeze as an entity and the heterogeneity of response to ICS and LTRA among children with preschool wheeze, ${ }^{6}$ evident even in relatively homogeneous RCT populations. ${ }^{9}$ In addition, because preschool wheeze is often self-limiting, spontaneous improvement over time could reduce the study power to detect an impact of the interventions. As reported in the "Results" section, in each of the eight cohorts, the proportions of children experiencing one or more wheezing/asthma attacks fell from the baseline year to the outcome year.

Our findings suggest that watchful waiting, obviously in conjunction with as-needed symptom management, may be the best approach for many children with preschool wheeze. This approach, however, should not preclude the assessment of several clinical factors that could suggest responsiveness to ICS or LTRA - especially for children who have evidence of symptoms between more severe episodes - in particular blood eosinophil count and aeroallergen sensitization, as well as other factors included in the modified API, namely physician-diagnosed atopic dermatitis, parental history of asthma, allergic sensitization to milk, eggs, or peanuts, and wheezing unrelated to colds. ${ }^{9}{ }^{9,4}{ }^{15}$ Fitzpatrick et al ${ }^{9}$ found that daily ICS was most likely to be efficacious for preschool children who had evidence of type 2 inflammation (aeroallergen sensitization and elevated blood eosinophil count). In a recent population-based study, ${ }^{3}$ allergic rhinitis, eczema, and parental asthma were three readily assessed variables associated with asthma diagnosis by 4 years of age in children with ever-wheeze. Finally, outcomes in another recent study suggested that children with the 5/5 ALOX5 promoter genotype may be responsive to intermittent montelukast 
therapy $(P$-value for interaction $=0.08) .{ }^{19}$ We were not able to assess these factors, as they were not evaluated in practice and/or recorded in the database for most children; however, an analysis including children with evidence of type 2 inflammation (aeroallergen sensitization and elevated blood eosinophil count or FeNO) is an important topic for further study in future observational studies using stratified analyses based on atopic status.

Prior work has suggested that early suppression of lung growth resulting in reduced maximum lung function in adulthood is associated with an increased risk of developing COPD. ${ }^{33-37}$ Childhood wheezing, childhood asthma, and childhood respiratory infections have been identified as factors associated with permanently lower lung function. ${ }^{33,35}$ While neither ICS nor LTRA therapy was associated with reduced odds of wheezing/asthma attacks over 1 year in this study, we cannot exclude the potential for long-term benefits of therapy on the trajectory of lung growth for some children, and hence a future reduction in the risk of adult conditions such as COPD.

We observed improvements across most end points, and for all treatment options, between baseline and outcome years. There could have been transient response to treatment in subgroups of children, but we speculate that for many children the greatest driver of improvement was likely time rather than treatment. Wheezing in young children can result from viral infections causing swelling and narrowing of small airways, a condition that does not respond to ICS but may be less likely to occur in subsequent years as the lungs grow in size.

In our study, ICS therapy was not associated with lower odds of wheezing/asthma attacks over the outcome year as compared with either SABA or LTRA therapy. There could in theory have been some dilution of comparative effects because $50 \%$ of children in the two SABA cohorts and $63 \%$ and $69 \%$ of those in the two LTRA cohorts were prescribed an ICS during the outcome year. However, we found no significant difference between matched cohorts for the time to first wheezing/asthma attacks after censoring children who had treatment failure (ie, change in therapy) before their first attack. Nevertheless, a pragmatic trial of patients who persist on their index date therapy could further elucidate this point.

In the LTRA vs ICS comparison, the fact that $69 \%$ of the children in the LTRA cohort were prescribed ICS in the outcome year while only $8 \%$ of the ICS cohort were prescribed LTRA during the outcome year suggests that those in the LTRA cohort had poorer control during the outcome year. Alternatively, this finding could be attributed to common prescribing pathways, whereby clinicians may be more likely to prescribe ICS to children whose symptoms do not respond to an LTRA than the converse (ie, prescribe LTRA to children whose symptoms do not respond to an ICS). Overall, many more children in the database were initiated on ICS $(n=11,329)$ than LTRA $(n=335)$.

Our findings are consistent with previous data and conclusions that maintenance controller therapy is not effective in managing episodic wheezing in preschool children. ${ }^{38-40}$ This study enabled us to record the occurrence of wheezing/asthma episodes over a 2-year time span, longer than most clinical trials. Most of the study cohorts were large, and cohort pairs were well-matched for baseline characteristics, as indicated by few remaining differences in baseline variables not used for matching. However, many eligible patients were excluded during the matching process, often at the last step when unique matched pairs were randomly selected and duplicate pairs were excluded; therefore, the cohort numbers were substantially reduced, limiting study power. Indeed, $<9 \%$ of ICS-treated children were included in the ICS/SABA analysis, and the two LTRA cohorts were relatively small (259 and 104 children), which could have limited statistical power to detect differences in outcomes. Moreover, some characteristics of the LTRA cohort as compared with the matched SABA cohort indicated more active disease at baseline (more antibiotics, ED visits, and repeat doses of OCS), although the only statistically significant differences were for ED visits and pneumonia, affecting few children and not always reliably recorded in general practice records. In addition, the matched LTRA/SABA cohorts were slightly younger than the matched ICS/SABA cohorts (mean 2.6 vs 3.2 years), which could have influenced the findings in light of the time effects. Overall, our study population may represent children on the milder end of the spectrum, as the study design required excluding children prescribed ICS before the age of 1 and those prescribed ICS or LTRA during the baseline year.

Another study limitation is that we were unable to ascertain whether children had persistent symptoms or were symptom-free between wheezing/asthma attacks. Moreover, the occurrence of adverse effects is not reliably recorded in medical records, hence not in the datasets, whereas a comparison of adverse occurrence associated with each treatment option would be useful information and constitutes an important area of further study. In addition, we could not assess medication adherence, and our study design could not distinguish between daily or intermittent ICS administration nor whether new therapy during the outcome year constituted a switch or add-on to existing therapy. The analyses depended on the quality of data recorded in primary care records, and 
the ability to clinically characterize the children was restricted to data available in their medical records. For example, we used an ever-recorded diagnosis of eczema, present for $38 \%-41 \%$ of children in each cohort, as an indicator of atopy. Other limitations include those common to all observational studies, including the potential for selection bias and unmeasured confounding factors. Moreover, it is likely that not all secondary care and hospital attendance information was captured in the longitudinal, electronic medical records housed at each individual's primary care practice. Although prescribing information in UK electronic medical records is generally considered reliable, ${ }^{41}$ we cannot be certain that medications were taken as prescribed.

We found high levels of antibiotic prescribing, with $50 \%-70 \%$ of children in each cohort prescribed at least one course of antibiotics during the baseline year and 35\%-45\% of children prescribed at least one course during the outcome year. The most recent UK pediatric audit (November 2015) found that $28 \%$ of preschool children presenting to hospitals and EDs with acute asthma/wheeze were prescribed antibiotics. ${ }^{4}$ These findings suggest that antibiotics are frequently prescribed for preschool wheeze despite the fact that most intermittent wheezing episodes are thought to be attributable to viral infections and therefore not responsive to antibiotics. ${ }^{10}$ Results of two studies have suggested that azithromycin therapy may decrease the length of episodes of lower respiratory symptoms in preschool children; ${ }^{42,43}$ however, further study is needed before determining whether widespread use of azithromycin for preschool wheeze is appropriate. ${ }^{44}$

\section{Conclusion}

This large matched cohort analysis of anonymized UK medical record data found no evidence that stepping up therapy, compared with as-needed use of SABA, reduces wheezing/ asthma attacks in a diverse population of preschool children with at least two documented prior wheezing episodes. Our findings underscore the need for better understanding of disease patterns and better targeting of existing therapies, or new therapeutic modalities, as well as the need for real-world data on adherence, atopic status, and emerging biomarkers. In the absence of better tools to help target treatments, and in light of improved outcomes over time in all cohorts, these data suggest that a "wait-and-see approach" for many preschool children with intermittent disease may be a clinically prudent approach.

\section{Abbreviations}

API, asthma predictive index

ED, emergency department

EF, extrafine
ICS, inhaled corticosteroid

$\mathrm{IQR}$, interquartile range

LABA, long-acting $\beta$-agonist

LTRA, leukotriene receptor antagonist

MMAD, mass median aerodynamic diameter

OCS, oral corticosteroid

pMDI, pressurized metered-dose inhaler

$\mathrm{RCT}$, randomized controlled trial

SABA, short-acting $\beta$-agonist

\section{Acknowledgments}

The authors gratefully acknowledge Ronan Ryan, $\mathrm{PhD}$, and Jaco Voorham, PhD (Observational and Pragmatic Research Institute Pte Ltd, Singapore), for statistical expertise and assistance with the analyses. This work was supported by the Respiratory Effectiveness Group (http://effectivenessevaluation.org/). Access to data from the Optimum Patient Care Research Database (http://optimumpatientcare.org/ opcrd/) was provided as an in-kind donation from Optimum Patient Care.

Collaborators from the Respiratory Effectiveness Group (REG) Small Airways Working Group and Child Health Working Group: Willem MC van Aalderen: Department of Pediatric Respiratory Medicine and Allergy, Emma Children's Hospital AMC, Amsterdam, the Netherlands. Clare S Murray: Division of Infection, Immunity and Respiratory Medicine, Manchester Academic Health Science Centre, The University of Manchester, University Hospital of South Manchester, and Royal Manchester Children's Hospital, Manchester, UK. Wanda Phipatanakul: Division of Allergy and Immunology, Boston Children's Hospital, and Harvard Medical School, Boston, MA, USA. Samatha Sonnappa: Department of Respiratory Paediatrics, Rainbow Children's Hospital, Bengaluru, India, and Observational and Pragmatic Research Institute Pte Ltd, Singapore, Singapore. Teoh Oon Hoe: Department of Paediatrics, Respiratory Medicine Service, KK Women's and Children's Hospital, Singapore, Singapore. Richard J Martin: Department of Medicine, National Jewish Health, and University of Colorado Denver, Denver, CO, USA. Alberto Papi: Department of Respiratory Medicine, University Hospital S. Anna, Ferrara, Italy. Stanley J Szefler: Breathing Institute, Children's Hospital Colorado, University of Colorado School of Medicine, Aurora, CO, USA. Derek Skinner: Optimum Patient Care, Cambridge, UK. R Brett McQueen: University of Colorado Skaggs School of Pharmacy and Pharmaceutical Sciences, Aurora, CO, USA. Omar S Usmani: Airway Disease Section, National Heart and Lung Institute, Imperial College London, and Royal Brompton Hospital, London, UK. 


\section{Author contributions}

JG, AN, AC, and DBP participated in the conception and design of the study. AN participated in the acquisition and analysis of the data. EVH developed the first draft of the manuscript. All authors participated in the interpretation of the data, the review and revision of the manuscript, and the approval to submit, and all agree to be accountable for all aspects of the work.

\section{Disclosure}

JG has received honoraria from GSK and Vifor Pharmaceuticals for Advisory Board membership. AN is an employee of the Respiratory Effectiveness Group. TWG reports personal fees from American Board of Pediatrics/Pediatric Pulmonary Subboard, personal fees from Teva, personal fees from GSK, personal fees from Regeneron Pharmaceuticals, grants from the National Institutes of Health (NIH), other from UpToDate, personal fees from Merck, personal fees from Sanofi, personal fees from Novartis, and personal fees from Aviragen. AK reports board membership with Aerocrine, AstraZeneca, Boehringer Ingelheim, Novartis, and Teva Pharmaceuticals, consultancy agreements with AstraZeneca, Boehringer Ingelheim, GlaxoSmithKline, Mylan, Novartis, Pfizer, Teva Pharmaceuticals, and Johsnson and Johnson, Purdue, grants and unrestricted funding for investigator-initiated studies from Novartis, payment for lectures/speaking engagements from AstraZeneca, Boehringer Ingelheim, Mylan, Merck, Novartis, Pfizer, Purdue Pharmaceuticals, and payment for the development of educational materials from Benton Dickinson. NR has received over the past 3 years fees for speaking, organising education, participation in advisory boards or consulting from 3M, AstraZeneca, Boehringer Ingelheim, Chiesi, Cipla, GlaxoSmithKline, MSD-Chibret, Mundipharma, Novartis, Pfizer, Sanofi, Sandoz, Teva, and Zambon, and research grants from Novartis, Boehringer Ingelheim, and Pfizer. EVH is a consultant to the Observational and Pragmatic Research Institute Pte Ltd (Singapore) and has received payment for medical writing work from Merck. DBP has board membership with Aerocrine, Amgen, AstraZeneca, Boehringer Ingelheim, Chiesi, Mylan, Mundipharma, Napp, Novartis, and Teva Pharmaceuticals, reports consultancy agreements with Almirall, Amgen, AstraZeneca, Boehringer Ingelheim, Chiesi, GlaxoSmithKline, Mylan, Mundipharma, Napp, Novartis, Pfizer, Teva Pharmaceuticals, and Theravance, grants and unrestricted funding for investigator-initiated studies (conducted through Observational and Pragmatic Research Institute Pte Ltd) from Aerocrine, AKL Research and Development Ltd, AstraZeneca, Boehringer Ingelheim,
British Lung Foundation, Chiesi, Mylan, Mundipharma, Napp, Novartis, Pfizer, Respiratory Effectiveness Group, Teva Pharmaceuticals, Theravance, UK National Health Service, and Zentiva, payment for lectures/speaking engagements from Almirall, AstraZeneca, Boehringer Ingelheim, Chiesi, Cipla, GlaxoSmithKline, Kyorin, Mylan, Merck, Mundipharma, Novartis, Pfizer, Skyepharma, and Teva Pharmaceuticals, payment for manuscript preparation from Mundipharma and Teva Pharmaceuticals, payment for the development of educational materials from Mundipharma and Novartis, payment for travel/accommodation/meeting expenses from Aerocrine, AstraZeneca, Boehringer Ingelheim, Mundipharma, Napp, Novartis, and Teva Pharmaceuticals, funding for patient enrollment or completion of research from Chiesi, Novartis, Teva Pharmaceuticals, and Zentiva, and stock/stock options from AKL Research and Development Ltd which produces phytopharmaceuticals, owns $74 \%$ of the social enterprise Optimum Patient Care Ltd (Australia, Singapore, and UK) and 74\% of Observational and Pragmatic Research Institute Pte Ltd (Singapore), and is a peer reviewer for grant committees of the Efficacy and Mechanism Evaluation programme and Health Technology Assessment. The other authors report no conflicts of interest in this work.

\section{References}

1. Bisgaard H, Szefler S. Prevalence of asthma-like symptoms in young children. Pediatr Pulmonol. 2007;42(8):723-728.

2. Martinez FD, Wright AL, Taussig LM, Holberg CJ, Halonen M, Morgan WJ. Asthma and wheezing in the first six years of life. The Group Health Medical Associates. N Engl J Med. 1995;332(3):133-138.

3. Uphoff EP, Bird PK, Antó JM, et al. Variations in the prevalence of childhood asthma and wheeze in MeDALL cohorts in Europe. ERJ Open Res. 2017;3(3)

4. British Thoracic Society. BTS National Paediatric Asthma Audit Summary Report (1-30 November 2015). 2016. Available from: https://www. brit-thoracic.org.uk/document-library/audit-and-quality-improvement/ audit-reports/bts-paediatric-asthma-audit-report-2015/. Accessed June 26, 2018.

5. Zein JG, Udeh BL, Teague WG, et al. Impact of age and sex on outcomes and hospital cost of acute asthma in the United States, 2011-2012. PLoS One. 2016;11(6):e0157301.

6. Beigelman A, Bacharier LB. Management of preschool children with recurrent wheezing: Lessons from the NHLBI's asthma research networks. J Allergy Clin Immunol Pract. 2016;4(1):1-8.

7. Ducharme FM, Tse SM, Chauhan B. Diagnosis, management, and prognosis of preschool wheeze. Lancet. 2014;383(9928):1593-1604.

8. Kaiser SV, Huynh T, Bacharier LB, et al. Preventing exacerbations in preschoolers with recurrent wheeze: a meta-analysis. Pediatrics. 2016;137(6):e20154496.

9. Fitzpatrick AM, Jackson DJ, Mauger DT, et al. Individualized therapy for persistent asthma in young children. J Allergy Clin Immunol. 2016;138(6):1608-1618.

10. British Thoracic Society, Scottish Intercollegiate Guidelines Network. British guideline on the management of asthma: a national clinical guideline. Revised 2016 (SIGN 153); 2016. Available from: http://www. sign.ac.uk/sign-153-british-guideline-on-the-management-of-asthma. html. Accessed June 26, 2018. 
11. Global INitiative for Asthma. 2016 GINA report, global strategy for asthma management and prevention; 2016. Available from: http:// ginasthma.org/archived-reports/. Accessed June 26, 2018.

12. Depner M, Fuchs O, Genuneit J, et al. Clinical and epidemiologic phenotypes of childhood asthma. Am J Respir Crit Care Med. 2014;189(2): 129-138.

13. van Wonderen KE, Geskus RB, van Aalderen WM, et al. Stability and predictiveness of multiple trigger and episodic viral wheeze in preschoolers. Clin Exp Allergy. 2016;46(6):837-847.

14. Castro-Rodríguez JA, Holberg CJ, Wright AL, Martinez FD. A clinical index to define risk of asthma in young children with recurrent wheezing. Am J Respir Crit Care Med. 2000;162(4 Pt 1):1403-1406.

15. Guilbert TW, Morgan WJ, Krawiec M, et al. The Prevention of Early Asthma in Kids study: design, rationale and methods for the Childhood Asthma Research and Education network. Control Clin Trials. 2004;25(3):286-310.

16. Guilbert TW, Morgan WJ, Zeiger RS, et al. Long-term inhaled corticosteroids in preschool children at high risk for asthma. $N$ Engl J Med. 2006;354(19):1985-1997.

17. Bacharier LB, Phillips BR, Zeiger RS, et al. Episodic use of an inhaled corticosteroid or leukotriene receptor antagonist in preschool children with moderate-to-severe intermittent wheezing. JAllergy Clin Immunol. 2008;122(6):1127-1135.

18. Zeiger RS, Mauger D, Bacharier LB, et al. Daily or intermittent budesonide in preschool children with recurrent wheezing. $N$ Engl J Med. 2011;365(21):1990-2001.

19. Nwokoro C, Pandya H, Turner S, et al. Intermittent montelukast in children aged 10 months to 5 years with wheeze (WAIT trial): a multicentre, randomised, placebo-controlled trial. Lancet Respir Med. 2014;2(10):796-803.

20. Robertson CF, Price D, Henry R, et al. Short-course montelukast for intermittent asthma in children: a randomized controlled trial. Am J Respir Crit Care Med. 2007;175(4):323-329.

21. Lenney W, Perry S, Price D. Clinical trials and tribulations: the MASCOT study. Thorax. 2011;66(6):457-458.

22. Price D, Bateman ED, Chisholm A, et al. Complementing the randomized controlled trial evidence base. Evolution not revolution. Ann Am Thorac Soc. 2014;11 Suppl 2:S92-S98.

23. Roche N, Reddel HK, Agusti A, et al. Integrating real-life studies in the global therapeutic research framework. Lancet Respir Med. 2013;1(10):e29-e30.

24. Chen W, Marra CA, Lynd LD, Fitzgerald JM, Zafari Z, Sadatsafavi M. The natural history of severe asthma and influences of early risk factors: a population-based cohort study. Thorax. 2016;71(3):267-275.

25. van Aalderen WM, Grigg J, Guilbert TW, et al. Small-particle inhaled corticosteroid as first-line or step-up controller therapy in childhood asthma. J Allergy Clin Immunol Pract. 2015;3(5):721-731.

26. Optimum Patient Care Research Database (OPCRD). Available from: http://opcrd.co.uk/. Accessed June 26, 2018.

27. Roche N, Reddel H, Martin R, et al. Quality standards for real-world research. Focus on observational database studies of comparative effectiveness. Ann Am Thorac Soc. 2014;11(Suppl 2):S99-S104.
28. Respiratory Effectiveness Group (REG). Available from: http://www. effectivenessevaluation.org/. Accessed June 26, 2018.

29. Reddel HK, Taylor DR, Bateman ED, et al. An official American Thoracic Society/European Respiratory Society statement: asthma control and exacerbations: standardizing endpoints for clinical asthma trials and clinical practice. Am J Respir Crit Care Med. 2009;180(1): 59-99

30. Colice G, Martin RJ, Israel E, et al. Asthma outcomes and costs of therapy with extrafine beclomethasone and fluticasone. J Allergy Clin Immunol. 2013;132(1):45-54.

31. Price D, Martin RJ, Barnes N, et al. Prescribing practices and asthma control with hydrofluoroalkane-beclomethasone and fluticasone: a real-world observational study. J Allergy Clin Immunol. 2010;126(3):511-518.

32. Sonnappa S, McQueen B, Postma DS, et al. Extrafine versus fine inhaled corticosteroids in relation to asthma control: A systematic review and meta-analysis of observational real-life studies. JAllergy Clin Immunol Pract. 2018;6(3):907-915.

33. Svanes C, Sunyer J, Plana E, et al. Early life origins of chronic obstructive pulmonary disease. Thorax. 2010;65(1):14-20.

34. Lange P, Celli B, Agustí A, et al. Lung-function trajectories leading to chronic obstructive pulmonary disease. $N$ Engl J Med. 2015;373(2): 111-122.

35. Ma H, Li Y, Tang L, et al. Impact of childhood wheezing on lung function in adulthood: A meta-analysis. PLoS One. 2018;13(2): e0192390.

36. Rennard SI, Drummond MB. Early chronic obstructive pulmonary disease: definition, assessment, and prevention. Lancet. 2015;385(9979): 1778-1788.

37. McGeachie MJ, Yates KP, Zhou X, et al. Patterns of growth and decline in lung function in persistent childhood asthma. $N$ Engl J Med. 2016;374(19):1842-1852.

38. Wilson N, Sloper K, Silverman M. Effect of continuous treatment with topical corticosteroid on episodic viral wheeze in preschool children. Arch Dis Child. 1995;72(4):317-320.

39. McKean M, Ducharme F. Inhaled steroids for episodic viral wheeze of childhood. Cochrane Database Syst Rev. 2000;(2):CD001107.

40. Doull IJ. Limitations of maintenance therapy for viral respiratory infection-induced asthma. J Pediatr. 2003;142(2 Suppl):S21-S25.

41. Tannen RL, Weiner MG, Xie D. Use of primary care electronic medical record database in drug efficacy research on cardiovascular outcomes: comparison of database and randomised controlled trial findings. $B M J$. 2009;338:b81.

42. Bacharier LB, Guilbert TW, Mauger DT, et al. Early administration of azithromycin and prevention of severe lower respiratory tract illnesses in preschool children with a history of such illnesses: a randomized clinical trial. JAMA. 2015;314(19):2034-2044.

43. Stokholm J, Chawes BL, Vissing NH, et al. Azithromycin for episodes with asthma-like symptoms in young children aged 1-3 years: a randomised, double-blind, placebo-controlled trial. Lancet Respir Med. 2016;4(1):19-26.

44. Grigg J. Antibiotics for preschool wheeze. Lancet Respir Med. 2016;4(1):2-3
Journal of Asthma and Allergy

\section{Publish your work in this journal}

The Journal of Asthma and Allergy is an international, peer-reviewed open access journal publishing original research, reports, editorials and commentaries on the following topics: Asthma; Pulmonary physiology; Asthma related clinical health; Clinical immunology and the immunological basis of disease; Pharmacological interventions and
Dovepress

new therapies. This journal is included in PubMed. The manuscript management system is completely online and includes a very quick and fair peer-review system, which is all easy to use. Visit http://www. dovepress.com/testimonials.php to read real quotes from published authors. 